\title{
En primera persona: investigando mundos de los que somos partes
}

\section{Em primeira pessoa: investigando mundos dos quais somos parte}

\section{I nvestigating worlds we are part of, in first person}

\author{
Saúl I . Fuks* \\ Profesor Titular de Psicología Clínica, Departamento de Postgrado, Facultad de \\ Psicología, Universidad Nacional de Rosario, Rosario, Argentina
}

\begin{abstract}
RESUMEN
El escrito propone algunas reflexiones en torno a los desafíos e interrogantes que se le presentan a los investigadores cuando se arriesgan a abandonar la ilusión de seguridad de colocarse "por fuera" de las dramáticas de su investigación. Los enfoques metodológicos de corte participativo y cooperativo cuestionan la posibilidad y el sentido de protegerse en la neutralidad u objetividad a fin de conseguir rigor y fiabilidad e invitan a considerar el encuentro de investigación sobre otras bases. En este escrito, revisamos algunos de los presupuestos epistemológicos y las dificultades metodológicas y técnicas que se presentan al investigador cuando intenta envolverse en la co-construccion de la investigación, arriesgándose a considerar a los "sujetos" como co-investigadores. Asimismo, se revisan algunas consecuencias de esta mirada sobre la formación de investigadores y se insinúan algunos caminos para cuidar - a la vez - del rigor metodológico y de una ética relacional.
\end{abstract}

Palabras claves: Co-construcción, Participación, Reflexividad, Dialogo.

\section{RESUMO}

Esse trabalho propõe algumas reflexões acerca dos desafios e questões que se apresentam aos pesquisadores quando se arriscam a abandonar a ilusão de segurança de não estarem envolvidos nas tramas de suas investigações. Os enfoques metodológicos de tipo participativo e cooperativo questionam a possibilidade e o sentido de proteger-se na neutralidade ou objetividade a fim de alcançar rigor e confiabilidade e convidam a considerar o encontro entre "pesquisador/pesquisado" em outras bases. Nesse trabalho, revisamos alguns pressupostos epistemológicos e as dificuldades metodológicas e técnicas que se apresentam ao investigador ao tentar envolver-se na construção da investigação e considerar os "sujeitos" como partícipes dessa construção. Nesse sentido, buscamos discutir as conseqüências de tal perspectiva na formação de pesquisadores e indicamos alguns caminhos que articulam a um só tempo rigor metodológico e ética relacional.

Palavras-chave: Co-construção, Participação, Reflexividade, Diálogo. 


\begin{abstract}
This article proposes some reflections around the challenges and questions that are presented to researchers when they take the risk to abandoning the illusion of security coming from positioning "outside" of their research. Participatory and collaborative methodological approaches questioned the possibility and the sense to protect the neutrality or objectivity in order to achieve accuracy and reliability and invited to consider the research encounter on other bases. In this paper, we review the basis of some epistemological, methodological and technical difficulties confronted for the researcher when he tries to get involved in the co-construction of research, taking the risk of considered the "subjects" as co-researchers. It also reviews some implications of this view on the training of investigators and we suggest some ways to look for a relational ethics and the methodological conditions.
\end{abstract}

Keywords: Co-construction, Participation, Reflexivity, Dialog.

\title{
I ntroducción
}

El objetivo de la investigación ${ }^{1}$ que generó estas reflexiones, ha sido el intento de obtener información y generar hipótesis que nos permitieran profundizar el conocimiento de las complejidades de las conversaciones organizadas por "parejas" en momentos decisivos de su relación. En este artículo nos proponemos hacer un recorrido por algunas cuestiones metodológicas (interrogantes, discusiones y tomas de posición) que serán - simultáneamente- el telón de fondo y el basamento de la forma de abordar las "conversaciones" y los "materiales" que sustentaron el trabajo. Asumimos una posición epistemológica que supone la participación y compromiso del investigador como un aspecto central del proyecto y consideramos necesario transparentar dicha postura dado que a partir de ella y de nuestros presupuestos, hemos podido ir al encuentro de la comunidad de investigación de este proyecto. Desplegar acá (aunque sea sintéticamente) esa trama, es una manera de reconocer y dar un lugar legítimo a los "mundos de sentido" que tejieron este proceso.

\section{Lenguaje, instrumento o realidad?}

El lenguaje, los objetos que éste nombra, los saberes que configura y las "conductas expresivas" al decir de Goffman (1993) son aquellos elementos que nos permiten acceder al mundo subjetivo de la gente. A medida que se ha ido produciendo la integración de perspectivas históricas, sociológicas, psicológicas y culturales- también se fueron generando, para describir las vidas de los otros, nuevos recursos e instrumentos.

Este proceso de integración y complejización fue parte de un movimiento que autores como Rabinow y Sullivan (1988) designaran como de "retorno del círculo hermenéutico" o una vuelta del "círculo de significado". Las diferentes perspectivas dentro de ese movimiento 
confluyeron en el supuesto que las personas comprenden y explican sus vidas a través de la creación de "historias" o "relatos" estructurados mediante guiones, caracteres, tiempos y lugares.

Han pasado ya más de 20 años desde la aparición del trabajo fundacional de Sarbin (1986) Narrative Psychology y en ese lapso el enfoque narrativo continuó expandiendo su influencia y su presencia se ha hecho notar en las investigaciones de las últimas décadas. Autores como Polkinghorne (1995), describieron a la "narrativa" como una forma lingüística fundamental para poder desplegar la existencia humana en situación; Clandinin y Connelly (2004) sostuvieron que el proceso de reconstrucción de las historias que la gente vive acabó convirtiéndose en un instrumento de transformación poderoso, basándose en que las investigaciones que constataron que al desplegar una historia es abrirla a las posibilidades de cambio. Por esta vía, el investigador puede tener acceso a los soportes que dan sentido al mundo de las personas, a la "interpretación" que hacen de los hechos, a los modos de construcción de la realidad y a la forma en que las personas articulan sus "teorías" o sistemas explicativos para poder legitimar sus actos y sus modos de vivir. Asimismo, estos enfoques pusieron en evidencia la necesidad de una mejor comprensión de los sistemas de valores y creencias, y de los significados que las personas adscriben a sí mismas y a su mundo lo cual se encuentra condensado en los relatos que las personas organizan para contar su experiencia (MISHLER, 1986).

En la investigación narrativa actual, esas "historias" y "relatos" han dejado de ser considerados como un "material de trabajo" o "un dato" para devenir "relatos co-construídos" conjuntamente con los investigadores. Este deslizamiento tuvo lugar a partir del corrimiento del "investigador neutro" y el surgimiento de un investigador coparticipe en la construcción de los relatos, alguien que con sus encuadramientos, preguntas, silencios, miradas y la presencia de su comunicación no-verbal contribuye activamente en la construcción y dirección del relato. La conciencia de este corrimiento (RAPOPORT, 1970) posibilito explorar más profundamente el encuentro entre "investigadores" e "investigados" hasta llegar a formularlo como una acción colaborativa organizado en torno a interrogantes comunes y significativos (SHOTTER, 1993). En este articulo, pondremos a consideración algunas ideas, algunas intuiciones y algunas referencias de como esto se reflejo en una investigación en la que tratamos de comprender las conversaciones que las parejas organizaban cuando su relación amorosa se encontraba en una crisis vivida como definitoria. 


\section{Perspectivas metodológicas y modos de posicionarse frente al investigar}

En nuestra visión, la investigación tradicional no ha valorizado positivamente la participación activa de los investigados lo que dio por resultado en una epistemología dividida, con tendencia a separar al conocedor de lo conocido facilitando la emergencia de formas relacionales de tipo autoritarias o colonial, en las cuales el "investigador" toma todas las decisiones acerca del contenido, metodología y hallazgos, y los "sujetos" de investigación son tratados como objetos pasivos de estudio.

En los modelos participativos y colaborativos, en cambio, estas posiciones asimétricas y excluyentes tienden a ser reemplazadas por relaciones de cooperación basadas en iniciativas y regulaciones recíprocas, lo cual implica un "hacer juntos" como co-investigadores y co-sujetos, confluyendo en el interés y curiosidad por interrogantes que hacen sentido para todos los involucrados. La Investigación Participativa y la Investigación Apreciativa (REASON, 1998) han sido intentos ejemplares de "modos de investigar" basados en un alto nivel de participación de los investigadores sin descuidar por ello la calidad metodológica (FUKS, 2003). Considerando similitudes y diferencias entre la Investigación Acción (AR), la Investigación Participativa (PR) y la Investigación Acción Participativa (IAP) se destaca como característica distintiva de la Investigación Participativa en la expresa intención de centrarse en la creación de un contexto y un proceso en el cual el conocimiento emergente de esos espacios se despliegue como parte de la "teoría local" (GEERTZ, 1994) que impregna a los participantes.

Esta manera de construir investigación, necesariamente requiere de un alto grado de compromiso por parte de todos los participantes (incluyendo a los investigadores) debido al activo papel que se les propone, convocándolos a incidir directamente en la definición de los problemas, en la elección o reformulación de los medios para obtener datos, de las formas de analizarlos, en la modalidad de construcción de los resultados y en la elaboración de acciones acordes. En este enfoque, la investigación se vuelve participativa, cuando aquellos directamente afectados por ella influencian cuatro campos de decisión: definición del problema, elección del método, análisis de los datos, y utilización de los resultados. Como Steier (1991) postulaba, el investigador deviene un co-productor de conocimientos y los participantes en este proceso, coherentemente, tienden a considerarse como colegas (BELL, J.; CHENEY, G.; HOOTS, C.; KOHRMAN, E.; SCHUBERT, K.; STIDHAM, L.; TRAYNOR, S. 2004) ${ }^{2}$ en el sentido que, a través de un proceso dialógico investigadores y participantes aprenden juntos, lo cual legitima a este enfoque como una autentica forma de co-investigación. Para llegar a ser parte de un 
enfoque con estas características no basta con una formación teórica, el investigador necesita desarrollar la flexibilidad suficiente para acompañar el flujo del proceso sin intentar dirigirlo, abandonando la tendencia a controlar "cómo y dónde" surgirán los datos, y ubicando en su lugar la capacidad de reflexión en medio de la incertidumbre. Este cambio de posicionamiento - de ser posible - le permitirá adecuarse a las perturbaciones provenientes de los cambios del contexto y los caminos elegidos por los participantes y, sustentado en esta posición, podrá contribuir al proceso emergente de producción colaborativa de conocimiento.

En un recorrido afín, encontramos las propuestas del enfoque de Investigación Cooperativa (REASON; HERON, 1986; REASON, 1994; HERON; REASON, 2001) quienes destacaron también la importancia de la participación de todos en el proceso de investigar, apostando a revalorizar las capacidades de agenciamiento del ser humano, que consideraron poco reconocidas dentro de la investigación ortodoxa. A pesar de que estos autores ubicaran la originalidad de su enfoque al lugar que atribuían a la participación en la construcción del investigar, en nuestra opinión el mayor desafío que proponen es poner en evidencia el tipo de posicionamiento necesario,

\footnotetext{
[...] los co-investigadores necesitan desarrollar una forma particular de "conciencia" que hemos llamado subjetividad crítica (p.124)...un estado de conciencia en la que, no suprimimos nuestra experiencia subjetiva primaria, sino que aceptamos que en nuestro encuentro vivencial con nosotros mismos y nuestro mundo, están las raíces de todo conocer....Subjetividad crítica envuelve una atención autoreflexiva acerca de la "base" desde la cual uno está partiendo y eso está muy cerca de lo que Bateson (1972) describe como Aprendizaje III y que Kegan (1994) refiere a una conciencia de 4to orden. (REASON; HERON, 1986, p. 125)
}

Propuestas epistemológicas y metodológicas como estas orientaron la concepción y el diseño de la investigación que comentaremos en este trabajo. Además impactaron en la forma de encarar la formación de investigadores, ya que evidenciaron el tipo de competencias comunicacionales que necesitan ser entrenadas para poder participar como investigadores en proyectos de Investigación con estas características. Estamos haciendo referencia al tipo de competencias comunicacionales alejadas de las formas tradicionales de "preguntarespuesta" o de las "entrevistas abiertas", tales como el "fluir-enconversación" o la co-construcción de interrogantes reflexivos y apreciativos que permiten desplegar y desarrollar una subjetividad crítica.

En el mismo sentido, podemos asumir que tal nivel de compromiso y participación por parte de los investigadores diseña verdaderas 
comunidades de acción e interpretación (ROYCE, 1916) en las que el proceso de conversar deviene acción conjunta (SHOTTER, 1993) que desborda el mero intercambio de informaciones. Expandiendo el mapa habitual de investigación, la perspectiva de una comunidad de investigación aporta tantos mundos a ser conocidos como personas envueltas en el acto de conocer, invitándonos a incorporar una compleja heterogeneidad epistemológica como dimensión inherente a ese tipo de proceso. Es en ese sentido que la construcción de una comunidad de investigación emerge como producto de un sistema relacional dialógico, en el que los diferentes participantes contribuyen a explorar las alternativas que surgen y ponen "su" instrumento de investigación - la capacidad de sorpresa/curiosidad- a disposición de la comunidad.

La construcción de una posición de investigador con estas características se aleja de una formación académica tradicional y excede al entrenamiento metodológico habitual $y$, en nuestra visión, esta capacitación requiere diseñar un posicionamiento (DAVIES; HARRE, 1982) que implique poder sostener consistentemente la cocreación de los conocimientos mediante capacidad de sorpresa, apertura, tolerancia a la incertidumbre ambigüedad y confusión, y a los laberintos inherentes a una exploración conjunta de lo noconocido. Una capacitación de investigadores que incorpore habilidades ligadas a esas condiciones reflexivas requiere un cambio epistemológico en el sentido Batesoniano y necesita la construcción de un contexto posibilitador que permita el despliegue de los saberesen-acción (ARGYRIS, 1980; ARGYRIS; SCHÖN, 1989; SCHÖN, 1996). En un proyecto en que los participantes están invitados a explorar interrogantes perturbadores, esto demanda de un contexto en el cual las perspectivas que se tengan acerca de la realidad puedan ser visualizadas, posibilitando que las diferentes realidades se desplieguen, se superpongan y se informen las unas a las otras. Los marcos dialógicos/reflexivos, así construidos, serán transformadores (además de metodológicamente sustentables) ya que, participantes e investigadores se verán envueltos en conversaciones en las cuales los interrogantes propuestos perturbarán la visión de las cosas y del mundo, arrasando con las ilusiones de objetividad o neutralidad. En consecuencia, y como punto de partida, en nuestro proyecto de investigación asumimos que los "invitados" eran los "expertos" en sus propios discursos y que contaban con conocimientos emergentes de los mundos que habitan y, por tanto, las posibilidades de reflexionar acerca de la propia vida y producir conocimientos a partir de esa experiencia, terminan siendo inseparables. Lo que sostenemos, es una manera de entender la generación de conocimientos que promueve una forma respetuosa de exploración de esos saberes/vida, basada en un posicionamiento que no se restringa al cuidado de los 
instrumentos de investigación y de sus condiciones de fiabilidad (CRAWFORD; LEYBOURNE; ARNOTT, 2000).

\section{"En primera persona": compromiso y participación del investigador}

Las perspectivas descritas aportaron interesantes alternativas cuando intentamos abordar algunos obstáculos encontrados en un proyecto en que tratábamos de ampliar el conocimiento acerca de las conversaciones que organizan las "parejas" amorosas cuando su relación se encuentra en un punto crítico $^{3}$. En especial, su utilidad se hizo manifiesta al intentar formular "teoría social" (NOFSINGER, 1991) acerca de las relaciones de pareja, meta que encontramos difícil de encarar mediante enfoques respaldados solamente en encuestas y/o estudios experimentales, debido al riesgo de que esta vías de abordaje diluyeran la riqueza de las singularidades y particularidades de esos mundos relacionales (BERGER; LUCKMAN, 1986; GERGEN, 1996).

Estas perspectivas nos aportaron contextos de libertad (FUKS, 2003) que facilitaron la construcción de relaciones próximas y colaborativas ${ }^{4}$ entre los participantes promoviendo, tanto en "investigadores" como "investigados", la expansión de una curiosidad genuina, tanto como la exploración de ideas instigadoras, visiones integrativas, sentimientos complejos y detalles sorprendentes. En el proyecto mencionado las entrevistas co-construidas con las parejas, en las que eran invitadas a explorar reflexivamente interrogantes ${ }^{5}$ respecto de sus mundos relacionales, estaban sostenidas en un marco que permitía que las diferentes visiones acerca de la realidad (o realidades) pudieran desplegarse y superponerse sin caer en disyunciones dilemáticas. Como punto de partida supusimos, que los entrevistados eran "expertos" en los mundos que habitaban, en las maneras de describirlos y en la identificación de los conocimientos que pudieran emerger de las experiencias relatadas. En consecuencia, asumimos que los procesos de reflexión acerca de las narrativas construidas y los conocimientos surgidos de esas experiencias constituían procesos inseparables; lo que propone una manera de entender el sentido de la investigación basado en una respetuosa forma de exploración que no se limite al rigor metodológico, al cuidado de los instrumentos de investigación y sus condiciones de fiabilidad (REASON; BRADBURY, 2001)

Los contextos de diálogos reflexivos (ALVENSSON; SKÓLDBERG, 2000) construidos sobre esta base, convocarán al investigador a una forma de participación poco aséptica o no-neutral y devienen (además de metodológicamente sustentables) en acciones potencialmente transformadoras ya que, en un escenario como el descrito, los participantes se verán interpelados por los interrogantes 
propuestos y serán (también) de-construidos mediante una forma de reflexión-en-acción que perturbará su visión de la vida y de los mundos relacionales en los que participan. Se trata de una concepción del diseño de investigación (TIETEL, 2000) que conecta con las propuestas de Investigación Apreciativa tanto como con las posiciones de no-saber y de curiosidad respetuosa propuestas por las psicoterapias posmodernas de raigambre sistémica. (ANDERSEN, 1994; ANDERSON; GOOLISHIAN, 1990, 1994, 1994; FUKS, 2000, 2004; TOMM, 1987, 1988).

Estos diseños poseen complejidades que impregnan el modo de concebir la "investigación" y la posición del investigador, una de ellas es que se necesita poder contar con una solida ecología social para el proyecto, necesaria para poder contrastar las conclusiones, así como del sostén de interlocutores capaces de organizar auditorios abiertos, flexibles y curiosos (BLAIR, 1998; BARRETT, 2005). En un diseño de investigación clásico (WIESSENFELD, 2000) los artilugios metodológicos serán el reaseguro imprescindible para validar las conclusiones a las que se pudiera arribar; en el tipo de enfoque que acá adoptamos esos resguardos provienen de los diálogos con la "comunidad de investigación" del proyecto, complejizados por las redes conversacionales de cada uno de los participantes lo que va tejiendo una trama organizada alrededor de los interrogantes que convocan y que delimitan el foco de la confluencia.

La propuesta de comunidad de investigación (BERGOLD, 2000) es otra manera de describir un círculo hermenéutico construido alrededor de interrogantes con energía suficiente como para despertar el interés, la curiosidad y la necesidad de participación, en línea con lo que Stanley Fish denominó comunidad de interpretación (FISH, 1976) En este sentido, las puertas de entrada a los intercambios posibles dependerán más de las conexiones singulares y microculturales con la temática, que con roles sociales ${ }^{6}$ preexistentes. Esto, implica una revisión de los perfiles tradicionales de sujeto, investigador, experto, etc. los que tienden a quedar relativizados debido a que el contexto y el flujo de la conversación serán los que acaben delimitando los espacios posibles y las formas de participación pertinentes. En nuestro trabajo, representamos esta comunidad mediante el modelo de la "margarita" propuesto por Barnett Pearce (1994; 2001) donde mediante la metáfora de una flor describe una trama en la que cada pétalo iconiza un campo conversacional construido por la densidad de sentidos que son aportados por cada uno de los involucrados sumados a los emergen en ese foco de conversación. En consecuencia, este proceso fue imaginado como un entrelazamiento de conversaciones (EPSTON, 1993) construidas en torno de los interrogantes organizadores de su foco. Hablamos de una trama de conversaciones expansible, expandida y expansiva (una 
red de diálogos) que contiene la complejidad adicional de intentar construir narrativas que sean respetuosas de la multivocalidad de los interlocutores. Congruentemente, la manera de tratar a los interrogantes fundantes de la investigación; la elección de quienes serán interlocutores privilegiados o circunstanciales, las reglas/normas generadas para el proceso y el tipo de argumentación que las volverán válidas son signos/actos que identificarán a la propuesta.

En función de lo descrito, consideramos a nuestro intento como parte de las corrientes comprometidas con la producción de prácticas liberadoras que rechazan el colonialismo que impregna aquellos diseños en los que el investigador entra en el mundo de los otros utilizando estrategias características de un poder colonizador (FUKS, 2007a). En las posiciones llamadas poscoloniales, los participantes del estudio son informados de manera transparente acerca de las metas de la investigación así como de la metodología propuesta, y son convocados a una activa participación en la construcción del contexto y del diseño, siendo convidados a legitimar sus voces, perspectivas e interpretaciones y a posicionarlas como un aspecto central de la investigación, configurando un proceso que trasciende el momento de los encuentros y se expande a la totalidad de la relación.

\section{Desafíos del análisis y la producción de resultados: co- construyendo "datos"}

En el mundo de los otros, inicialmente oscuro, polisémico y sin un sentido evidente para el investigador, este deviene -así- en el otro de los otros (MORIN, 1994b) un extraño, un extranjero (FUKS, 2007b) e carece de claves para entender el sentido de esos relatos y para quién comienzan a emerger significados, en la medida en que sus constructores comparten con él las vías para acceder a una comprensión. Nuestro mundo cotidiano está cada vez más lleno de información la que está disponible bajo formas diferentes $y$, sin embargo, no todas las informaciones son significativas, ni tienen la misma relevancia y jerarquía para nosotros. Lo que las vuelve relevantes y las destaca del conjunto de otras informaciones (diferentes o similares) es la forma en que se conectan con nuestro "interés", o con aquello que "necesitamos" saber.

En los comienzos de la era de la informática, Gregory Bateson lanzó una de las ideas que aún hoy continúan orientando nuestra concepción sobre el "conocer" (VARELA, 1990). Bateson (1972) propuso que toda información relevante es información acerca de una diferencia y, a partir de ahí, en la producción de conocimiento el lugar de las informaciones cambió, dejaron de ser consideradas como elementos aislados que podían ser tratados con la lógica cartesiana y comenzaron a ser consideradas como ingredientes particularizados de 
una "ecología" sociocultural. En esta perspectiva, la dimensión comunicativa ha dejado de ser considerada como un mero instrumento, como un "medio" (GERGEN, 1996), y devino la dimensión donde la cultura se hace sujeto-en-acto (IBAÑEZ, 2004); para comprender tal densidad, el investigador necesita construir una relación de estar adentro - en conexión - y a la vez, fuertemente ligado a sus preguntas e interrogantes (MORIN, 1994a) en especial con aquellas que lo condujeron a participar en el proyecto.

\section{Las herramientas: la "entrevista reflexiva"}

En modelos metodológicos que cuidan la calidad de los resultados en base a la creencia que el investigador puede protegerse detrás de los artilugios técnicos, las herramientas técnicas son meros instrumentos que se pueden ajustar para volverlos "universales", que pueden ser utilizados por cualquier investigador si se respetan ciertas pautas (SCHÖN, 1987). ¿Qué tipo de instrumento podría ser útil en un enfoque donde la persona del investigador está tan profundamente comprometida en el proceso de investigar? A fin de encarar este desafío, desarrollos provenientes del campo de la psicoterapia y de los enfoques de facilitación sistémica de procesos colectivos, aportan la posibilidad de pensar diseños conversacionales para la generación de marcos reflexivos que, al mismo tiempo, puedan atender al rigor metodológico necesario para que el conocimiento producido pueda ser incorporado como patrimonio de una comunidad científica (ATKINSON; CHENAIL, 1991).

En sintonía con los enfoques que promueven la construcción de conversaciones colaborativas, al investigar las conversaciones de parejas co-construimos una forma de entrevista exploratoria a la carte (FUKS, 2008). Desde la perspectiva del entrevistador, dichas entrevistas fueron sustentadas en una posición de exploración reflexiva y de "no saber", y se diseñaron tomando en cuenta las maneras de participar en conversaciones mediante preguntas, formulaciones/reformulaciones, y elección léxica más cercanas a las psicoterapias que a los métodos de investigación. El tipo de exploración ${ }^{7}$ al que nos estamos refiriendo fue construido integrando formatos utilizados por investigadores cualitativos (TIAINEN; KOIVUNEN 2006), las experiencias y conocimientos como psicoterapeuta de parejas, intentando alcanzar una confluencia epistemológica, teórica metodológica y técnica congruente.

En el diseño de la investigación, el propósito principal de estas entrevistas (basadas en la exploración ${ }^{8}$ y la curiosidad reflexiva) estuvo orientado a la facilitación de un dialogo colaborativo más que a la obtención de informaciones y datos. En la tradición sistémica, este tipo de entrevistas reflexivas han sido consideradas como formas de "conversaciones colaborativas" expresamente orientadas a 
incrementar la comprensión (FELDMAN, 1995) que transcurren en un marco de cuidadosa negociación de las diferencias entre descripciones, explicaciones, interrogantes y visiones alternativas, donde cada interlocutor puede tener la oportunidad de hablar y de ser escuchado respetuosamente. En consecuencia, explorar es una manera de circular en un mundo de significados posibles y - por tanto - las preguntas que construimos/compartimos podrán funcionar como "instrumentos de navegación" que orientarán, algunas veces ayudarán a distinguir entre opciones y otras abrirán aún más la complejidad del panorama. No obstante, no todas las exploraciones podrán desplegar esa cualidad, ni todas las preguntas (independientes del contenido) cooperarán con ese tipo de viaje, el investigador requiere de ciertas "competencias de exploración" 9 para crear condiciones que hagan posible que se produzca. La cualidad de la que hablamos es una producción emergente de un contexto; no se trata de algo "inherente" o "esencial" a una forma de preguntar, sino al modo en que forma, contenido y contexto se articulan en un diseño (FUKS, 2000).

La forma en que el entrevistador sostiene las preguntas con foco en la relación, abre posibilidades de hablar de un modo diferente. La forma tanto como el marco creado a las preguntas las envuelven de un tono reflexivo, curioso e interesado, que permite un hablar que excederá el intercambio de informaciones ${ }^{10}$ y que puede ser incorporado a la cultura ${ }^{11}$ de la entrevista como método (GUBA, 1990). Este marco, habilita y autoriza al intento de desnaturalizar las palabras claves del relato ${ }^{12}$; en un proceso que generará innovación no sólo para el investigador sino para todos los participantes quienes, en este marco, se escucharán de un modo distinto ${ }^{13}$ (FERNANDOIS, 2008). El poder constructor/deconstructor de las metáforas abre una valiosa vía de acceso a las multiplicidades de sentidos que aparecen condensados en los nudos complejos de los relatos (SMITH, 1981) debido a ello la emergencia de metáforas en el flujo de los intercambios facilita la inmersión en contenidos que necesitan ser tratados con cuidado, sosteniendo "en acto" la propuesta de coinvestigar. En nuestra visión, como fue siendo propuesto a lo largo de este trabajo, revisar y deconstruir la posición del investigador, su manera de construir los datos, los modos de interactuar con esos datos, la construcción de herramientas singulares y el diseño dialógico mediante el cual ese complejo sistema hace emerger conocimiento, es una de las aventuras que nos propone la artesanía de investigar. 


\section{Bibliografía}

ALVENSSON, M.; SKÓLDBERG, K. Reflexive methodology: new vistas for qualitative research. London: Sage, 2000.

ANDERSEN, T. El equipo reflexivo. Diálogos y diálogos sobre diálogos. Buenos Aires: Ed. Gedisa, 1994.

ANDERSON, $H_{.} ;$GOOLISHIAN, H. El experto es el cliente: la ignorancia como enfoque terapéutico. En: MCNAMEE, S.; GERGEN, K. J. (Eds.). La terapia como construcción social. Buenos Aires: Paidos, 1996, p. 45-61.

. Human systems as linguistic systems. Evolving ideas about the implications for theory and practices. Family process.27 (4). 371393.1988.

Narrativa y self. Algunos dilemas posmodernos de la psicoterapia. In: SCHNITMAN, D. F. (Ed.). Nuevos paradigmas, cultura y subjetividad. Buenos Aires: Paidos, 1994.

ARGYRIS, C. Inner contradictions of rigorous research. New York: Academic Press, 1980, p.25-39.

ARGYRIS, C.; SCHÖN, D. A. Participatory action research and action science compared: a commentary. American behavioral scientist, v.32, p. 612-623, 1989.

ATKINSON, B. H. A.; CHENAIL, R. Qualitative research and the legitimization of knowledge. Journal of marital and family therapy, v. 17, p. 175-180, 1991.

BARRETT, F.; FRY, R. Appreciative inquiry: a positive approach to building cooperative capacity. Chagrin Falls, Ohio: Taos Institute Publications, 2005.

BATESON, G. Steps to an ecology of mind. New York: Ballantine Books, 1972.

BELL, J.; CHENEY, G.; HOOTS, C.; KOHRMAN, E.; SCHUBERT, J.; STIDHAM, L.; TRAYNOR, S. Comparative similarities and differences between action research, participative research and participatory action research. Critical inquiry. Chicago: Univ. of Chicago Press, Summer 2004.

BERGER, P. L.; LUCKMAN, T. La construcción social de la realidad. Buenos Aires: Amorrortu, 1986.

BERGOLD, J. B.: The affinity between qualitative methods and Community Psychology. Forum Qualitative Sozialforschung, v.1, n.2, J une 2000. C

BLAIR, M. Lessons from the field: applying appreciative inquiry. Medford, OR: Practical Press. Inc., 1998.

CLANDININ, D. J.; CONNELLY, F. M. Narrative inquiry: experience and story in qualitative research. Califórnia: John Wiley; Sons Inc. 2004.

CRAWFORD, H. K; LEYBOURNE, M. L.; ARNOTT, A. How we ensured rigour in a multi-site, multi-discipline, multi-researcher study. Forum 
qualitative sozialforschung [On-line Journal], v.1, n.1, jan. 2000. (2000, January).

DAVIES, B.; HARRE, R. Positioning: the discursive production of selves. In: The TAOS Institute. The virtual faculty. 1982. Disponível em: <http://www.massey.ac.nz>. Acesso em: set.2009.

EPSTON, D. Internalizing vs externalizing discourse en therapeutic conversations. In: GILLIGAN S.; PRICE, R. (Eds). New York: Norton. N.Y., 1993, p. 161-177.

FELDMAN, A. Conversation as research: a self-study of the teaching of collaborative action research. Paper presented the Annual Meeting of AERA, San Francisco, CA., April 18-22, 1995. FERMANDOIS, F. Wittgenstein, Geertz y la comprensión de metáforas. Critica. Rev. Hispanoamericana de Filosofía, v.40, n. 118, p. 29-56, abr. 2008.

FISH, S. Interpreting the variorum. Chicago: Critical Inquirí, 1976. FUKS, S. I. Transformando las conversaciones acerca de las transformaciones. Educación, salud y trabajo. Revista I beroamericana (Coedición Universidad Nacional de Rosario y Universidad de Extremadura), n.1, p. 213-232, abr. 2000.

. (colab) La investigación acción participativa y el debate epistemológico en el marco de las incertidumbres postmodernas. Rivarola: T. Publ. del Postgrado de Gestión en Servicios Sociales / Univ. Complutense de Madrid, Setiembre 2003, p 15-20.

Craftsmanship of contexts; an unfinished story of my connection with CMM. In: Guest Editors, W. Barnett Pearce and Jeremy Kearney. Human Systems. Special Edition: CMM. Extensions and Applications. London: KCC Ed. v.15, 2004, p. 1-3. . Estabilidad y Cambio en las relaciones de pareja. 2006. 437.pag. Tesis Doctoral (Doctorado en Psicología Clínica) - Univ. de Belgrano, Buenos Aires.

- Reflexiones acerca de la paradoja del empowerment. In: SAAFÖORCADA, E.; CERVONE, N.; CASTELLÁ SARRIERA, J.; LAPALMA, A. y DE LELLIS, M. Aportes de la psicología comunitaria a problemáticas de la actualidad latinoamericana. Buenos Aires: JVE ediciones, 2007-a, p. 19-50.

Ecos: O eventual, o banal e as conexões transformadoras. Nova perspectiva sistêmica, ano XVI, n.29, p.126-129, 2007-b. A relação de casal como organização social: desafios e oportunidades. Nova Perspectiva Sistémica, ano XVI, n.30, p.2144, 2008.

GEERTZ, C. Conocimiento local. Buenos Aires: Paidos. 1994. GERGEN, K. Realidad y relaciones. Aproximaciones a la construcción social. Buenos Aires: Paidos, 1996. 
GOFFMAN, E. La presentación de la persona en la vida cotidiana. Buenos Aires: Amorrortu, 1993.

Narrativa y self. Algunos dilemas posmodernos de la psicoterapia. In: SCHNITMAN, D. F. (Ed.). Nuevos paradigmas, cultura y subjetividad. Buenos Aires: Paidos, 1994.

GUBA, E. G. The paradigm dialog. Newbury Park, Ca: Sage, 1990. HERON, J.; REASON, P. The practice of co-operative inquiry: research with rather than on people. In: REASON, P.; BRADBURY, H. (Eds.). Handbook of action research: participative inquiry and practice. London: Sage, 2001, p.179-188.

IBÁÑEZ, Jesús. El regreso del sujeto. La investigación social de segundo orden. España: Siglo XXI, 1994.

MORIN, E. Epistemología de la complejidad. In: SCHNITMAN, D. F. (Ed.). Nuevos paradigmas, cultura y subjetividad. Buenos Aires: Paidos, 1994-a, p. 421-423.

MORIN, E. La noción de sujeto. In: SCHNITMAN, D.F. (Ed.) Nuevos paradigmas cultura y subjetividad. Buenos Aires: Paidos, 1994-b, p. 67-86.

MISHLER, E. Research interviewing: context and narrative. Cambridge: Harvard Press, 1986.

NOFSINGER, R. E. Everyday conversations. Newbury Park, CA: Sage, 1991.

PEARCE, W.B. Nuevos modelos y metáforas comunicacionales: el pasaje de la teoría a la praxis, del objetivismo al construccionismo social y de la representación a la reflexividad. In: SCHNITMAN, D. F. (Ed.). Nuevos paradigmas, cultura y subjetividad. Buenos Aires: Paidos, 1994, p. 265-284.

- Introducción a la teoría del manejo coordinado del significado.

Sistemas familiares, v.17, n. 2, p.130-146, 2001.

POLKINGHORNE, D. E. Narrative knowing and the human sciences. Albany, NY: State of New York University Press, 1988.

RABINOW P.; SULLIVAN W. M. (Ed.). I nterpretive social science. A second look. California: Univ. of Calif. Press, 1988.

RAPOPORT, R. N. Three dilemmas in action research. Human relations, v. 33, n.6, p. 499-513, 1970.

REASON, P. (Ed.). Participation in human inquiry. Newbury Park, CA: Sage, 1994.

REASON, P.; HERON, J. Research with people: The paradigm of cooperative experiential inquiry. Person Centred Review, v.1, p. 456475, 1986.

REASON, P; BRADBURY, $\mathrm{H}$. Conclusion: broadening the bandwidth of validity: issues and choice-points for improving the quality of action research. In: REASON, Peter; BRADBURY, H. (Eds.). Handbook of action research. Participatory inquiry and practice. Thousand Oaks, CA.: Sage, 2001, p. 447-55. 
REASON, P. Three approaches to participative inquiry. In: Denzin Norman K. ; Lincoln Yvonna S. (Eds.) Strategies of qualitative inquiry. Thousand Oaks: Sage, 1998, p. 261-291.

ROYCE, J. The hope of the great community. Nova York: Macmillan Ed. NY., 1916.

SARBIN, T. R. (Ed.). Narrative psychology: the storied nature of human conduct. New York: Praeger, 1986.

SCHÖN, D. A. Educating the reflective practitioner: toward a new design for teaching and learning in the professions. San Francisco: J ossey-Bass, 1987.

SCHÖN, D. A. Educating the reflective practitioner: toward a new design for teaching and learning in the professions. San Francisco: J ossey-Bass, 1987.

SCHÖN, D. La crisis del conocimiento profesional y la búsqueda de una epistemología de la práctica. In: PAKMAN, M. Construcciones de la experiencia humana. (Comp.). v. I. Barcelona: Gedisa, 1996, p. 183-187.

SHOTTER, J. Conversational realities: Constructing life through language. Thousand Oaks, CA: Sage Pub, 1993.

SMITH, N. L. (Ed.). Metaphors for evaluation: sources of new methods. Beverly Hills, CA: Sage, 1981.

STEIER, F. Research and reflexivity. London: Sage, 1991. TIETEL, E. The interview as a relational space. Forum qualitative sozialforschung, v.1, n.3, dec. 2000. http://www.qualitativeresearch.net

TIAINEN, T.; KOIVUNEN, E. R. Exploring forms of triangulation to facilitate collaborative research practice: reflections from a multidisciplinary research group. Journal of Research Practice, v.2, n.2, article 2006. http://jrp.icaap.org/index.php/jrp/issue/view/4

TOMM, K. Interventive interviewing: Part II. Reflexive questioning as a means to enable self-healing. Family process, v. 26, p. 167-184, 1987.

Interventive interviewing: Part III. Intending to ask lineal, circular, strategic, or reflexive questions. Family process, v.27, p.115, 1988

VARELA, F. Conocer: las ciencias cognitivas tendencias y perspectivas. Cartografía de las ideas actuales. Barcelona: Gedisa, 1990.

WIESENFELD, Esther. Between prescription and action: the gap between the theory and practice of qualitative inquiries [65 paragraphs]. Forum qualitative sozialforschung [On-line Journal], v.1, n.2. Disponível em: http://qualitative-research.net/fqs/fqs-e/2o0inhalt-e.htm Acesso em: ago. 2009. 


\section{Endereço para correspondência}

Saúl I. Fuks

Wheelwright 1711, Piso 9 - 2000, Rosario, Santa Fe, Argentina

Endereço eletrônico: cocofuks@gmail.com

Recebido em: 17/08/2009

Aceito para publicação em: 17/09/2009

Acompanhamento do processo editorial: Deise Mancebo, Marisa Lopes da Rocha e Roberta Romagnoli.

\section{Notas}

* Doctor en Psicología Clínica. Universidad de Belgrano. Buenos Aires. Argentina.

1 Fuks, S. I. Estabilidad y Cambio en las relaciones de pareja. Tesis Doctoral. Universidad de Belgrano. Buenos Aires. Argentina. 2006.

${ }^{2}$ En nuestro proyecto hablábamos de co-investigadores.

3 “¿En la experiencia de Uds., qué ayuda a poder conversar y salir enriquecidos como pareja- cuando se está en una situación donde existe la posibilidad de terminar con la relación?"

4 en el cual la construcción del contexto de investigación dista de las "técnicas" tradicionales para conseguir la "colaboración" de los sujetos y requiere -por el contrario- de una co-participación activa y comprometida.

5 “¿En la experiencia de Uds., qué ayuda a poder conversar y salir enriquecidos como pareja- cuando se está en una situación donde existe la posibilidad de terminar con la relación?"

${ }^{6}$ Esto vuelve a este tipo de intercambios algo cercano a la manera en que Edgar Morin definió la producción transdisciplinaria del conocimiento.

7 Dentro de la expresión genérica "explorar" incluimos la manera de construir el contexto de la entrevista (tales como el posicionamiento del investigador, el tipo de convocatoria) así como la forma de las preguntas, formulaciones y elecciones léxicas utilizadas en la propuesta de co-investigar un determinado aspecto o una forma de vida.

${ }^{8}$ Preferimos hablar de exploración en lugar de interrogatorio o preguntas ya que consideramos que la forma de hacer preguntas es sólo un aspecto de la exploración.

9 Capacidad para construir contextos; invitar al dialogo, negociar reglas para ese espacio; escuchar; preguntar, responder, co-construir, describir, reformular; metaforizar y legitimar.

10 Tal como B. Pearce et al propusieron, las conversaciones organizan (y son organizadas) por sistemas de reglas acerca de lo que se debe o se prohíbe, tanto en cuanto al "acceso" como en cuanto a los "derechos", en este caso la relación doctor/paciente asociada a saber/no-saber, se transforma en un modo de funcionamiento mas participativo.

11 "Cultural" en el sentido de Geertz, cuando plantea que la cultura es una comunidad de aprendices que cooperan para dar sentido al "mundo" que comparten.

12 La metáfora de las palabras claves de los hiperlinks, que resaltan del resto del texto (no por su contenido sino por la forma, color, subrayado y signos que indican su condición de "algo que remite a otra cosa") encaja con la manera propuesta de posicionarse respecto a la densidad polisémica de las conversaciones. Abrir una "palabra" (expresión idiomática, noción, imagen, etcétera) que invita al interlocutor a presuponer una comprensión, puede ser tanto una "amenaza", como una navegación en el mundo de sentidos. La obtención de los passwords, en una 
relación que intenta ser colaborativa, es parte de la construcción minuciosa que tiene a las preguntas y al modo de tratar a las contextualizaciones como browsers.

${ }^{13}$ Concretando uno de los requisitos de la reflexividad: posicionarse frente a si mismo como otro. 